\title{
Current developments in ion mobility spectrometry
}

\section{Gérard Hopfgartner ${ }^{1}$ (D)}

Received: 9 July 2019 / Accepted: 9 July 2019 /Published online: 30 July 2019

(C) Springer-Verlag GmbH Germany, part of Springer Nature 2019

Since the early work of J.J. Thompson to determine the mass of the electron, more than 100 years ago, mass spectrometry (MS) has established itself as a key technique in analytical science. Almost any analyte (e.g., element, chemical, pharmaceutical, metabolite, protein) can be analyzed by MS on the basis of its massto-charge ratio $(\mathrm{m} / \mathrm{z})$ assuming that positively or negatively charged particles can be formed during ionization. Multiple analytes can be separated in different low-resolution or highresolution mass analyzers because of their specific behavior $(\mathrm{m} /$ $z)$ in an electric or magnetic field. Another unique feature of charged molecules is their specific motion in an electric field in the presence of a neutral gas, used today in ion mobility spectrometry (IMS). IMS separates positively or negatively charged analytes on the basis of the size-to-charge ratio. This is referred to as the collisional cross section (CCS), which is specific for each compound and gas. Compared with MS, IMS operates mostly at atmospheric or reduced pressure and is ideally suited for the development of small portable and inexpensive devices. In recent decades, IMS has evolved as a stand-alone technique for ambient analysis in airport and transportation security focusing on the detection of explosives and illicit drugs. Many other applications have been reported, including air quality analysis, food control, medical diagnostics, process control, environmental analysis, and forensic analysis. IMS and MS have been developed independently, but for the analysis of complex samples both techniques will likely not be sufficient either for unambiguous analyte identification or for precise and accurate quantification. IMS has limited resolution, whereas MS suffers from limited differentiation of isomeric or isobaric analytes. The "hyphenation" of separation methods (e.g., gas chromatography or liquid chromatography) with MS is successful because of the orthogonality of both

Published in the topical collection Close-Up of Current Developments in Ion Mobility Spectrometry with guest editor Gérard Hopfgartner.

Gérard Hopfgartner

gerard.hopfgartner@unige.ch

1 Life Sciences Mass Spectrometry, Department of Inorganic and Analytical Chemistry, University of Geneva, 24 Quai Ernest Ansermet, 1211 Geneva 4, Switzerland techniques, and the hyphenation of IMS and MS is an obvious approach as $m / z$ and mobility are complementary features. For a very long time IMS has been the playground of academic laboratories using systems developed in-house. Nowadays, most MS system vendors have implemented IMS in their instrumentation, opening new exiting analytical possibilities regarding the analysis of complex samples or for high-throughput analysis. Many different forms of IMS coexist, including two major approaches: (1) drift time IMS (DTIMS) and (2) differential IMS (DMS), also referred to as "field asymmetric waveform ion mobility spectrometry" (FAIMS). DTIMS and DMS take advantage of the differences in the mobility of ions in high electric fields. DMS and FAIMS are generally coupled with low-resolution MS in selected-reaction-monitoring mode and act as a filter to improve quantification. DTIMS, when coupled with use of high-resolution mass analyzers allows the generation and use of CCS libraries to improve analyte identification. This topical collection focusing on ion mobility presents many features of IMS additional to those previously mentioned and will provide readers with some valuable insights into recent developments and applications of IMS.

I thank all authors for their contributions and the reviewers for their time and efforts to make this collection possible.

Publisher's note Springer Nature remains neutral with regard to jurisdictional claims in published maps and institutional affiliations.

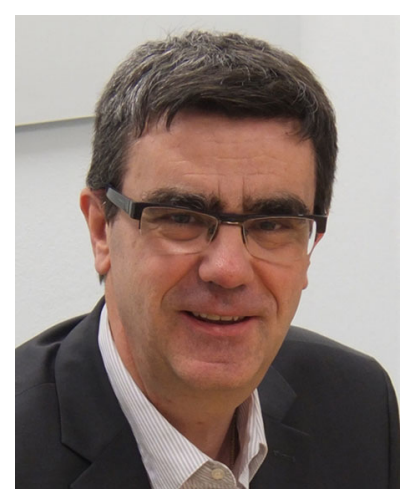

Gérard Hopfgartner is Professor of Analytical Chemistry and Mass Spectrometry in the Department of Analytical and Inorganic Chemistry of the University of Geneva. His research interests focus on the development and application of novel hyphenated mass spectrometry in the field of life sciences. 